\title{
Critical electronic structures controlling phase transitions induced by lithium ion intercalation in molybdenum disulphide
}

\author{
CHEN XiaoBo, CHEN ZhenLian \& LI Jun ${ }^{*}$ \\ Ningbo Institute of Material Technology \& Engineering, Chinese Academy of Sciences, Ningbo 315201, China
}

Received December 19, 2012; accepted February 17, 2013

We report on first-principles studies of lithium-intercalation-induced structural phase transitions in molybdenum disulphide $\left(\mathrm{MoS}_{2}\right)$, a promising material for energy storage in lithium ion batteries. It is demonstrated that the inversion-symmetry-related Mo-S $p$ - $d$ covalence interaction and the anisotropy of $d$-band hybridization are the critical factors influencing the structural phase transitions upon $\mathrm{Li}$ ion intercalation. $\mathrm{Li}$ ion intercalation in $2 \mathrm{H}-\mathrm{MoS}_{2}$ leads to two competing effects, i.e. the $2 \mathrm{H}$-to- $1 \mathrm{~T}$ transition due to the weakening of Mo-S $p-d$ interaction and the $D_{6 h}$ crystal field, and the charge-density-wave transition due to the Peierls instability in Li-intercalated $2 \mathrm{H}$ phase. The stabilization of charge density wave in Li-intercalated $\mathrm{MoS}_{2}$ originates from the enhanced electron correlation due to nearest-neighbor Mo-Mo $d-d$ covalence interaction, conforming to the extended Hubbard model. The magnitude of charge density wave is affected by Mo-S $p$ - $d$ covalence interaction and the anisotropy of $d$-band hybridization. In $1 \mathrm{~T}$ phase of Li-intercalated $\mathrm{MoS}_{2}$, the strong anisotropy of $d$-band hybridization contributes to the strong Fermi surface nesting while the $d$-band nonbonding with S- $p$ facilities effective electron injection.

$\mathrm{MoS}_{2}$, phase transition, charge density wave, $p$ - $d$ interaction, first-principles

Citation: Chen X B, Chen Z L, Li J. Critical electronic structures controlling phase transitions induced by lithium ion intercalation in molybdenum disulphide. Chin Sci Bull, 2013, 58: 1632-1641, doi: 10.1007/s11434-013-5834-y

Molybdenum disulphide $\left(\mathrm{MoS}_{2}\right)$ is increasingly important for a variety of applications in electronics [1-3], optics [4-6], hydrogen storage [7], catalysts [8,9], lubricants $[10,11]$, and double-layer capacitors [12]. The quasi-twodimensional structural characteristic facilitates lithium ion intercalation and extraction, analogous to that in graphite $[13,14]$. The much higher capacity of $\mathrm{MoS}_{2}(600-1200 \mathrm{~mA}$ $\mathrm{h} / \mathrm{g}$ ) than that of graphite/graphene (372-900 mA h/g) makes $\mathrm{MoS}_{2}$ an excellent candidate for fabricating highcapacity and rechargeable lithium ion batteries (LIBs) [15-26]. Clearly, the electrochemical performance of $\mathrm{MoS}_{2}$ is closely related to the structural phase transitions induced by $\mathrm{Li}$ ion intercalation [14,27]. This leads to the imperative need for understanding the mechanism of intercalationinduced phase transitions in order to realize its full electrochemical potential for energy storage.

It is commonly believed that upon $\mathrm{Li}$ ion intercalation

*Corresponding author (email: lijun@nimte.ac.cn)
$\mathrm{MoS}_{2}$ may undergo a $2 \mathrm{H}$-to-1T phase transition, in which the local $\mathrm{MoS}_{6}$ unit transforms from the original trigonal prism into an octahedron [28-31]. This belief is challenged by a recent in situ XRD result which indicates amorphization of $\mathrm{MoS}_{2}$ host, rather than the formation of 1T phase [32]. On the other hand, the first-principles calculations predict a Fermi-surface-driven charge density wave (CDW) in Li-intercalated $1 \mathrm{~T}-\mathrm{MoS}_{2}$ [33], which is featured by a Mo plane distortion with diamond pattern [33-36]. Another type of Mo plane distortion with zigzag-chain pattern is reported for single-layer and restacked $\mathrm{MoS}_{2}$ [37,38]. We have demonstrated in a recent calculation that the $\mathrm{CDW}$ formation in Li-intercalated $1 \mathrm{~T}-\mathrm{MoS}_{2}$ coincides with the maximization of chemical hardness of Li-intercalated $\mathrm{MoS}_{2}$ host [27]. However, ambiguity exists in understanding of the mechanisms of both $2 \mathrm{H}$-to-1T transition and CDW stabilization. Especially the issue concerning whether $\mathrm{Li}$ intercalated $2 \mathrm{H}-\mathrm{MoS}_{2}$ can undergo a CDW transition similar to that in the 1T counterpart have not been addressed. 
All these hamper the understanding of experimentally observed inconsistency and thus further improvements in performance. Additionally, although the electronic structures of $2 \mathrm{H}-$ and $1 \mathrm{~T}-\mathrm{MoS}_{2}$ and Li-intercalated compounds (e.g. $2 \mathrm{H}-$ and $1 \mathrm{~T}-\mathrm{LiMoS}_{2}$, and the CDW phase of $1 \mathrm{~T}-\mathrm{LiMoS}_{2}$ ) have been studied intensively [39-44], the critical electronic properties governing relative phase stability (with and without $\mathrm{Li}$ ion intercalation) and electron injection have not been revealed, imposing difficulties in uncovering the mechanisms of structural phase transitions.

The target of this theoretical work is twofold, first, to identify the critical electronic states near Fermi surface associated with phase stability and outer electron injection due to Li ion intercalation, and second, to analyze the mechanisms of intercalation-induced phase transitions of $\mathrm{MoS}_{2}$. It is demonstrated that the inversion-symmetry-related Mo-S $p$ - $d$ interaction and the anisotropy of $d$-band hybridization are the critical factors influencing the structural phase transitions. When Li ions are intercalated, $2 \mathrm{H}-\mathrm{MoS}_{2}$ may transit to $1 \mathrm{~T}$ phase due to the weakening of Mo-S $p$ - $d$ interaction and the $D_{6 h}$ crystal field. It is found that CDW exists not only in Li-intercalated $1 \mathrm{~T}-\mathrm{MoS}_{2}$ but also in the corresponding $2 \mathrm{H}$ counterpart, with a relatively weak magnitude in the latter due to the inversion-symmetry-related Mo-S $p$ - $d$ interaction. The CDW transition in Li-intercalated $2 \mathrm{H}-\mathrm{MoS}_{2}$ may compete with the $2 \mathrm{H}$-to-1T transition, perhaps leading to a structural disorder corresponding to the observed amorphization of $\mathrm{MoS}_{2}$ host. The stabilization of CDW in Li-intercalated $\mathrm{MoS}_{2}$ can be ascribed to the enhancement of electron correlation due to nearest-neighbor Mo-Mo $d$ - $d$ interaction.

\section{Structures and computational details}

$\mathrm{MoS}_{2}$ crystal consists of S-Mo-S tri-layer slabs held together by weak vdW force. Changes in the ion arrangement within an S-Mo-S slab may lead to two typical building blocks, i.e. trigonal prism and octahedron. Three polytypes, i.e. trigonal $1 \mathrm{~T}$, hexagonal $2 \mathrm{H}$, or rhombohedral $3 \mathrm{R}$, have been experimentally obtained. The structural models are representatively shown for $1 \mathrm{~T}$ and $2 \mathrm{H}$ phase in Figure 1 . In this work, initial unit-cells for these polytypes are constructed from experimental lattice parameters and internal coordinates [45-47].

Structural differences of these three polytypes are summarized in Table 1. Point groups of 1T, $2 \mathrm{H}$, and $3 \mathrm{R}$ phases are $D_{3 d}, D_{6 h}$, and $C_{3 v}$ respectively. Both $D_{3 d}$ and $D_{6 h}$ have inversion symmetry, while $C_{3 v}$ does not. For the $D_{3 d}$ point group of 1T phase, inversion centers are at Mo sites. For the $D_{6 h}$ point group of $2 \mathrm{H}$ phase, they are at interstitial octahedral sites within vdW gaps, even though the trigonal prismatic $\mathrm{MoS}_{6}$ unit has no inversion center.

Octahedral and tetrahedral interstitial sites for $1 \mathrm{~T}$ and $2 \mathrm{H}$ phase are shown in Figure 1. These are potential sites for Li

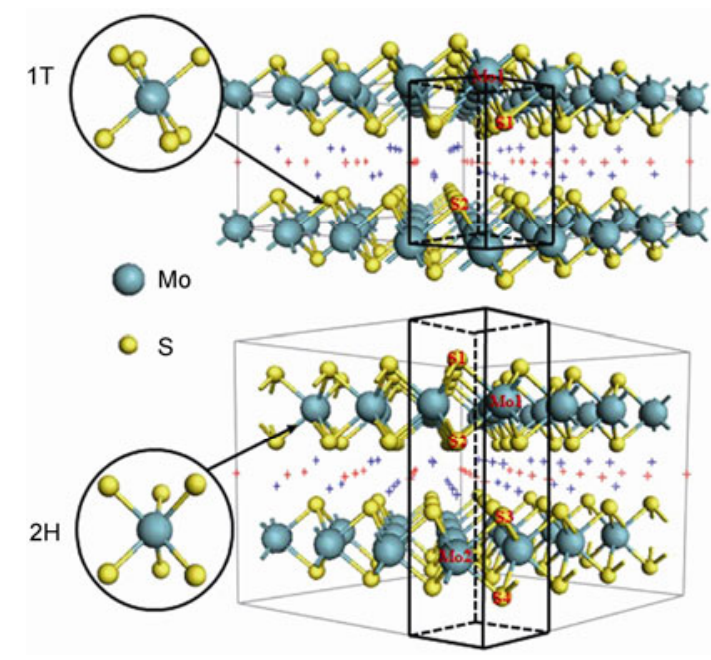

Figure 1 Perspective views of structural models and building blocks for $1 \mathrm{~T}$ - and $2 \mathrm{H}-\mathrm{MoS}_{2}$. The corresponding unit cells are indicated by the black-line boundaries containing atoms labeled with red symbols. Octahedral and tetrahedral interstitial sites in the vdW gap are represented by red and blue crosses, respectively.

ion intercalation. The number of tetrahedral sites is twice that of octahedral sites. Within a vdW gap, interstitial sites can be separated into three layers, with one octahedral interstitial layer sandwiched by two other tetrahedral interstitial layers. The three interstitial layers displace each other by one third of the diagonal line length in the (001) face.

Total energy calculations are performed within the framework of density functional theory (DFT), as implemented in the Vienna Ab-initio Simulation Package (VASP) [48]. Interactions of electrons with ion cores are represented by the projector augmented wave (PAW) potential [49]. A cutoff energy of $400 \mathrm{eV}$ is used for the plane-wave expansion of wave functions. Integrations over Brillouin zone use Monkhorst-Pack special $k$ point mesh of $18 \times 18 \times 4$ for the $2 \mathrm{H}$ and $3 \mathrm{R}$ unit-cells, and $18 \times 18 \times 8$ for the $1 \mathrm{~T}$ unit-cell, and $8 \times 8 \times 8$ for the unit cell of the CDW phase of $1 \mathrm{~T}-\mathrm{LiMoS}_{2}$ (denoted thereafter as $\mathrm{C} 1 \mathrm{~T}-\mathrm{LiMoS}_{2}$ ) [33] respectively. Structural relaxations are performed with all the forces acting on ions smaller than $0.005 \mathrm{eV} / \AA$. Energy uncertainties due to the selection of cutoff energy and $k$ point sampling are smaller than $0.002 \mathrm{eV} /$ ion. For pure $\mathrm{MoS}_{2}$, the vdW correction in Grimme's DFT-D2 scheme [50] is used for structural determination. This gives overall deviations smaller than $1.1 \%$ for $a, c$, and thickness $T$, and a deviation smaller than $2 \%$ for $\theta$ of S-Mo-S from the experimental values, as shown in Table 1. For Li-intercalated systems, we use only PBE for calculations because vdW interactions are expected to be suppressed by Li-host electrostatic interactions. As a comparison, optimized structural parameters for C1T-LiMoS ${ }_{2}$ are listed with other available theoretical and experimental values in Table 2. In general, our calculations agree well with the experiment of Mulhern [30]. We also test the effect of vdW correction and find that the prediction of $c$ is worsened remarkably relative to the result of pure 
Table 1 Structural and energy properties of $2 \mathrm{H}-, 3 \mathrm{R}$ - and $1 \mathrm{~T}-\mathrm{MoS}_{2}$ calculated at the GGA-PBE level corrected with van der Waals interactions ${ }^{\text {a) }}$

\begin{tabular}{llll}
\hline & \multicolumn{1}{c}{$2 \mathrm{H}$} & \multicolumn{1}{c}{$3 \mathrm{R}$} & \multicolumn{1}{c}{$1 \mathrm{~T}$} \\
\hline Stacking & $\mathrm{cBcbCb}$ & $\mathrm{cAcbCbaBa}$ & $\mathrm{cAb}$ \\
Point group & $D_{6 h}$ & $C_{3 v}$ & $D_{3 d}$ \\
$c l a$ & 3.887 & 5.799 & 1.872 \\
$d_{\text {Mo-s }}(\AA)$ & 2.416 & 2.416 & 2.430 \\
$d_{\text {Mo-Mo }}(\AA)$ & 3.196 & 3.198 & 3.225 \\
$d_{\text {S-s }}(\AA)$ & 3.119 & 3.116 & 3.637 \\
Inter-slab distance $(\AA))$ & 6.212 & 6.182 & 6.036 \\
Volume $\left(\AA^{3} /\right.$ f.u. $)$ & 54.940 & 54.755 & 54.351 \\
$E_{\text {tot }}(\mathrm{eV} /$ f.u. $)$ & -22.457 & -22.457 & -21.665 \\
$E_{1}(\mathrm{eV} /$ f.u. $)$ & -170.733 & -169.807 & -166.532 \\
$E_{2}(\mathrm{eV} /$ f.u. $)$ & 149.056 & 148.134 & 145.685 \\
$E_{\text {vdW }}(\mathrm{eV} /$ f.u. $)$ & -0.780 & -0.784 & -0.818 \\
\hline
\end{tabular}

a) $d_{\text {Mo-S }}$ and $d_{\text {Mo-Mo }}$ denote bond lengths of Mo-S and Mo-Mo respectively. $d_{\mathrm{S}-\mathrm{S}}$ denotes the inter-ionic distance of two nearest neighboring $\mathrm{S}$ ions bonding on different sides of Mo planes. The inter-slab distance indicates the separation between two neighboring Mo planes. In the line of 'stacking', capital letters means the positions of Mo and the lowercase the positions of S.

Table 2 Optimized cell parameters at the PBE level compared to the FLAPW results and the experimental one for C1T-LiMoS

\begin{tabular}{lccclll}
\hline & $a(\AA)$ & $b(\AA)$ & $c(\AA)$ & $\alpha\left({ }^{\circ}\right)$ & $\beta\left({ }^{\circ}\right)$ & $\gamma\left({ }^{\circ}\right)$ \\
\hline Exp. [28] & 6.720 & 6.720 & 6.294 & 90 & 90 & 120 \\
Exp. [30] & $6.75-6.80$ & $6.75-6.80$ & 6.26 & 90.0 & 90.0 & 121.2 \\
FLAPW [33] & 6.70 & 6.70 & 6.39 & 90 & 90 & 120 \\
This work & 6.85 & 6.80 & 6.28 & 90.13 & 90.10 & 121.43 \\
\hline
\end{tabular}

GGA. It is noted that the FPLAPW calculations [33] agree with the experiment of Py et al. [28] only in the prediction of $a$ and $b$, but not $c$. Therefore, it is not necessary to include vdW correction for $\mathrm{LiMoS}_{2}$. In our PBE optimization, the original hexagonal lattice distorts slightly into a triclinic one, resulting in a total energy reduction of $\sim 50 \mathrm{meV} / \mathrm{f}$.u.

\section{Results and discussion}

\subsection{Electronic properties: $p$ - $d$ interaction and $d$-band hybridization}

Band structures of relaxed $2 \mathrm{H}, 3 \mathrm{R}$, and $1 \mathrm{~T}$ phases are compared in Figure 2(a). $2 \mathrm{H}$ phase has an indirect band gap of $0.93 \mathrm{eV}$, smaller than the experimental one of $1.29 \mathrm{eV}$ [51], in agreement with previous DFT calculations [46,52]. The valence band maximum (VBM) is at $\Gamma$ and the conduction band minimum (CBM) lies midway between $\Gamma$ and K. Similar to $2 \mathrm{H}$ phase, $3 \mathrm{R}$ has an indirect fundamental band gap of $0.86 \mathrm{eV}$. However, the VBM is shifted from $\Gamma$ to $\mathrm{A}$ and the $\mathrm{CBM}$ is shifted from the midway of $\Gamma-\mathrm{K}$ to $\mathrm{H}$ due to the band folding effect induced by the increase of stacking periodicity of S-Mo-S slabs along the $c$ axis. In contrast to $2 \mathrm{H}$ and $3 \mathrm{R}$ phases, $1 \mathrm{~T}$ bands present the feature of metal, which is ascribed to the $D_{3 d}$ symmetry of $\mathrm{MoS}_{6}$ building blocks.

Mo- $4 d$ and S-3p constitute the major valence band of $\mathrm{MoS}_{2}$. Under $D_{6 h}$ point group, the five degenerate Mo- $4 d$ orbitals split into two doubly-degenerate states $e\left(d_{x z}, d_{y z}\right)$ and $e^{\prime}\left(d_{x y}, d_{x-y}^{2}{ }^{2}\right)$, and one singly degenerate state $a\left(d_{z}{ }^{2}\right)$, as shown in Figure 3. The latter two hybridize with each other due to the mirror reflection with respect to the $x$-y plane crossing Mo ions, resulting in the fundamental band gap in the $d$-band manifold and the energy reduction of the $a$-subband. The hybridized $a$-subband (mixing with $d_{x y}$, $d_{x-y}^{2}$, and $d_{z}^{2}$ ) can bond covalently with S-p to form a bonding $p-d$ state and an antibonding $(p-d)^{*}$ one, reducing the energy of occupied states. It is different for $D_{3 d}$ symmetry in that Mo- $4 d$ orbitals split into an $e_{g}$ state $\left(d_{x z}, d_{y z}\right)$ and a $t_{2 g}$ state $\left(d_{x y}, d_{x-y}^{2}, d_{z}^{2}\right)$. The bonding of $t_{2 g}$ to $S-p$ is forbidden because of the inversion symmetry centered at Mo sites.

The evolution of Mo-S $p$ - $d$ mixing in $\mathrm{MoS}_{2}$ lattice is presented in Figure 4. The squares of coefficients of each angular momentum wave functions for the highest occupied valence band of $2 \mathrm{H}$ - and $1 \mathrm{~T}-\mathrm{MoS}_{2}$ are extracted from the first-principles calculations respectively. They are summed up along the K- $\Gamma-\mathrm{M}$ route for S- $p$ and Mo- $d$, as shown in Figure 4(a) and (c). For $2 \mathrm{H}$ phase $p$ and $d$ can mix independent on $k$. For 1T phase, on the contrary, the mixing is forbidden at $\Gamma$ but weakly permitted for $k$ points away from $\Gamma$. It is obvious that the majorities of $p$ and $d$ are distributed in different parts, i.e. $p$ state is centralized around $\Gamma$, but $d$ state away from $\Gamma$. This inversion-symmetry-related $p$ - $d$ interaction indicates that covalent bonding is stronger in $2 \mathrm{H}$ phase than in 1T phase. In the following, it will be seen that Mo-S $p$ - $d$ interaction imposes significant effect on electron injection due to $\mathrm{Li}$ ion intercalation and the magnitude of CDW in Li-intercalated $\mathrm{MoS}_{2}$.

Figure 4(b) and (d) indicates the effect of $k$ dispersion on the $d$-state hybridization for the highest occupied valence band of $2 \mathrm{H}-$ and $1 \mathrm{~T}-\mathrm{MoS}_{2}$, respectively. In $2 \mathrm{H}$ phase, the $d$-state hybridization is formed by $d_{x y}, d_{x-y}^{2}$, and $d_{z}^{2}$, which are distributed nearly symmetrically at the two sides of $\Gamma$ except for the boundaries, indicating it is generally isotropic. In $1 \mathrm{~T}$ phase, in contrast, $k$ dispersion leads to anisotropic hybridization, i.e. the hybridization is changed from the original $\left(d_{x y}, d_{x-y}^{2}{ }^{2}, d_{z}^{2}\right)$ to orientation-dependent new combinations. This hybridization anisotropy also exists in lower valence bands, such as band B and C shown in Figure 2(a). It will be shown in the following that the anisotropy of $d$-state hybridization of edge bands determines the Fermi surface shape, which affects the CDW transition of Liintercalated $\mathrm{MoS}_{2}$.

The partial density of states (DOS) in Figure 2(b) show that the formation of band gap in $2 \mathrm{H}$ phase correlates indeed first to the strong hybridization between $d_{z}^{2}$ and $\left(d_{x y}, d_{x-y}^{2}{ }^{2}\right)$, and then to the strong Mo-S $p$ - $d$ covalence interaction that lowers the $a$-subband further. The energy reduction induced by both $d$-subband hybridization and $p$ - $d$ covalence 

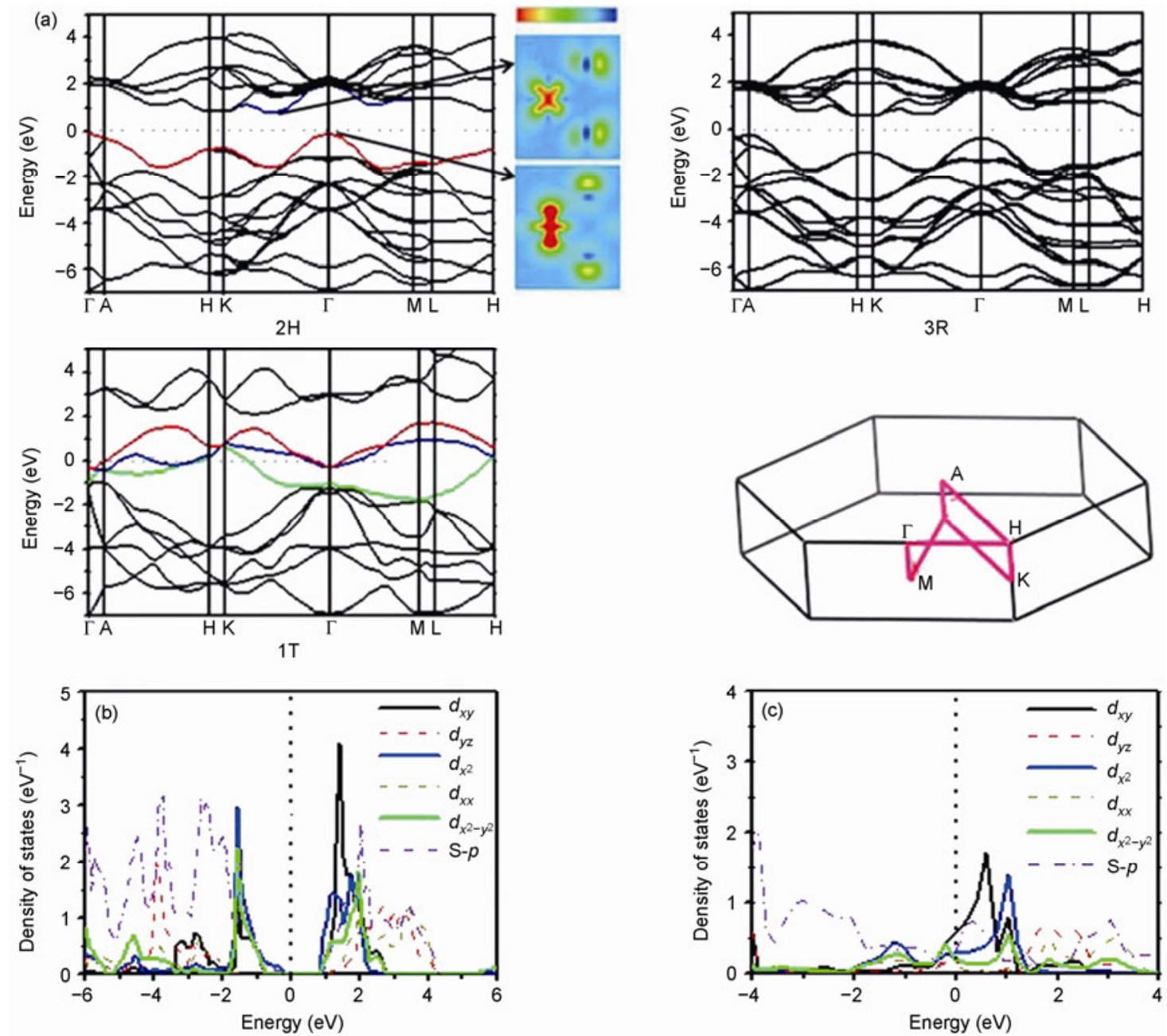

Figure 2 (a) Band structures of relaxed 2H-, 3R- and 1T- $\mathrm{MoS}_{2}$ along the high-symmetry points (connected by red lines) shown in the irreducible Brillouin zone of a hexagonal unit-cell. The Fermi energy is set to be energy zero point, denoted by dotted line. (110)-plane projected partial charge density for the valence band maximum and conduction band minimum of $2 \mathrm{H}$ phase are presented together. Charge density value decreases according to the color change from red to green and then to blue. (b) and (c) are the partial density of states of $2 \mathrm{H}$ - and $1 \mathrm{~T}-\mathrm{MoS}_{2}$, respectively.

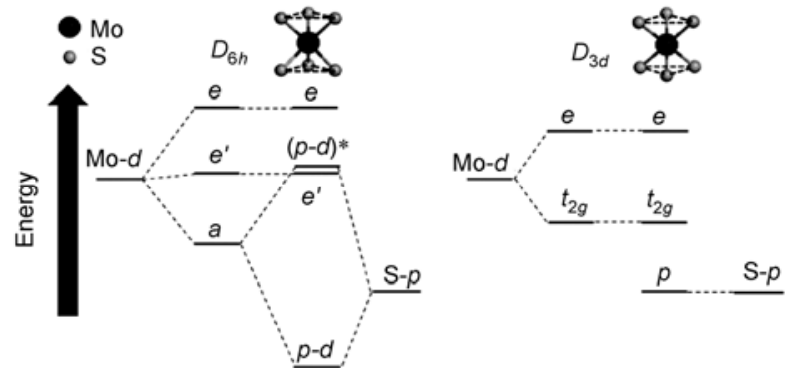

Figure 3 Energy-level diagram of molecular orbital model for $p-d$ interaction under $D_{6 h}$ group.

interaction stabilizes the $2 \mathrm{H}$ phase at ground state. Since the $4 d^{2}$ electronic configuration of $\mathrm{Mo}^{4+}$ leads to a full occupation of the lowest $a$-subband, $2 \mathrm{H}$ phase is a semiconductor. In the partial charge profiles in Figure 2(a), one can easily identify the $p$ - $d$ bonding characteristic of the VBM and the antibonding characteristic of the CBM. The antibonding part is overlapped in energy with the hybridized $e^{\prime}$-subband.
1T phase is metallic because the $t_{2 g}$-band remains weak bonding with S- $p$ and partially filled under the $4 d^{2}$ electronic configuration of $\mathrm{Mo}^{4+}$. Figure 2(c) shows that the $t_{2 g}$-band is constituted mainly by $d_{x y}, d_{x-y}^{2}{ }^{2}$ and $d_{z}^{2}$, rather than $d_{x y}, d_{y z}$, and $d_{x z}$ assumed previously [40]. The $e_{g}$-band is composed of $d_{x z}$ and $d_{y z}$. We find that in the $t_{2 g}$-band only $d_{x y}$ has substantial overlap with S- $p$ due to $k$ dispersion, in contrast to the case of $2 \mathrm{H}$ phase where $d_{x y}, d_{x-y}^{2}{ }^{2}$ and $d_{z}^{2}$ all have strong covalence with the latter. This accounts for the weak Mo-S $p-d$ covalence in 1T phase, leaving the electronic bands crossing the Fermi level determined primarily by the crystal-field. The Fermi surface is formed by three bands denoted by A, B, and C in Figure 2(a). The rising of A-band in the midway of both $\Gamma-\mathrm{M}$ and $\Gamma-\mathrm{K}$ is due to the fact that Mo- $4 d$ is higher in energy than S-3p.

\subsection{Phase stability of $\mathrm{MoS}_{2}$ polytypes}

Since different polytypes of $\mathrm{MoS}_{2}$ have the same element 

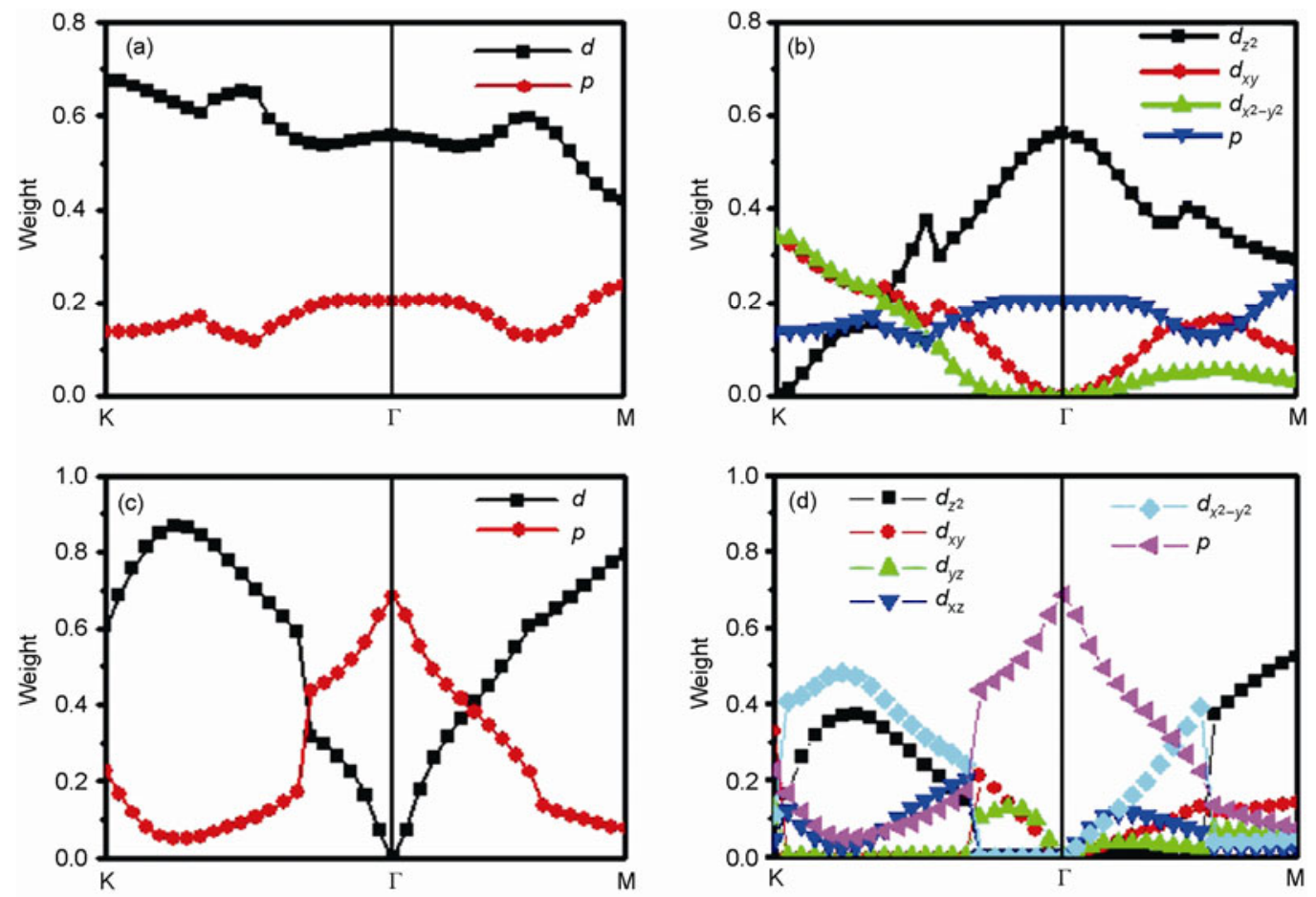

Figure 4 Coefficient squares of $p$ and $d$ orbitals along the path K-Г-M for the top valence band (shown by red lines in Figure 4) of $\mathrm{MoS}_{2}$. (a) and (b) are for $2 \mathrm{H}-\mathrm{MoS}_{2}$, and (c) and (d) for $1 \mathrm{~T}-\mathrm{MoS}_{2}$.

constitution and differ only in the symmetry, direct comparison of stability in terms of relative energy is possible because their cohesive energies can be referred to the same reference energy, i.e. the formula-unit (f.u.) total energy when all the atoms are separated infinitely. This is the standard total energy approach in the first-principles structure determination.

In normal state, $2 \mathrm{H}$ and $3 \mathrm{R}$ are more stable than $1 \mathrm{~T}$, as could be seen from the f.u. total energies $E_{\text {tot }}$ in Table 1 . This is natural because both $d$-subband hybridization and Mo-S $p$ - $d$ covalence in $2 \mathrm{H}$ and $3 \mathrm{R}$ can lower the total energies by lowering the occupation state energy, as indicated above. Under current computational accuracy, the energy stability is identical for $2 \mathrm{H}$ and $3 \mathrm{R}$. This indicates that stacking order is less important than slab symmetry in structural stabilization. To quantify the origin of stability, one can compare the different contribution to the KohnSham total energy [53] from

$$
E_{1}=\sum_{i} \varepsilon_{i}+\int n(\vec{r})\left\{\varepsilon_{x c}[n(\vec{r})]-\mu_{x c}[n(\vec{r})]\right\} \mathrm{d}^{3} r
$$

and

$$
E_{2}=-\frac{1}{2} \iint \frac{n\left(\overrightarrow{r_{i}}\right) n\left(\overrightarrow{r_{j}}\right)}{\left|\overrightarrow{r_{i}}-\vec{r}_{j}\right|} \mathrm{d}^{3} r_{i} \mathrm{~d}^{3} r_{j}-\frac{1}{2} \iint \frac{Z_{I} Z_{J} e^{2}}{\left|\overrightarrow{R_{I}-R_{J}}\right|} \mathrm{d}^{3} R_{I} \mathrm{~d}^{3} R_{J}
$$

$E_{1}$ is a sum of the total KS band energy and XC energy, an indication of the occupation state energy. $E_{2}$ presents the classic Coulomb repulsion energy, containing the electron- electron Hartree energy and the ion-ion Ewald energy only. Note that $E_{1}$ and $E_{2}$ are all finite number and their sums are the normal total energy by the first-principles methods. Therefore, one can use them for relative energy comparison between the polytypes. The vdW energy is defined as [50]

$$
E_{\mathrm{vdW}}=-\frac{s_{6}}{2} \sum_{i=1}^{N_{a L}-1} \sum_{j=i+1}^{N_{a t}} \frac{C_{6}^{i j}}{R_{i j}^{6}} f\left(R_{i j}\right),
$$

where parameters in eq. (3) are assigned according to [44]. As shown in Table 1, $E_{1}$ is higher by $\sim 0.93 \mathrm{eV} /$ f.u. for $3 \mathrm{R}$ than for $2 \mathrm{H}$. However, the difference of $E_{2}+E_{\mathrm{vdW}}$ between $2 \mathrm{H}$ and $3 \mathrm{R}$ cancels that of $E_{1}$ exactly, resulting in an identical energy stability of them. For $1 \mathrm{~T}$ phase, on the contrary, the relative rising magnitude of $E_{1}(4.201 \mathrm{eV} /$ f.u.) with respect to that of $2 \mathrm{H}$ is much larger than the relative decrease magnitude of coulomb energies (3.371 eV/f.u.) between them, leading to the lower stability of $1 \mathrm{~T}$ than $2 \mathrm{H}$ and $3 \mathrm{R}$. The large increase of $E_{1}$ is a result of the change of crystal-field splitting and the absence of Mo-S $p-d$ interaction due to the occurrence of inversion symmetry centered at Mo sites. It is clear that crystal-field stabilization is the major reason for the stability of $2 \mathrm{H}$ phase, which is reinforced by Mo-S $p$ - $d$ interaction.

\subsection{Phase stability upon $\mathrm{Li}$ intercalation: $2 \mathrm{H}-\mathrm{to}-1 \mathrm{~T}$ transition}

It is commonly believed that the $2 \mathrm{H}$-to- $1 \mathrm{~T}$ phase transition occurs at the $1.1 \mathrm{~V}$ voltage plateau of discharging curve 
[23,24,28]. During the transition, the ion ratio between $\mathrm{Li}$ and Mo changes continuously in the range of $(0,1)$. To model $\mathrm{Li}$ ion intercalation in this range, we construct a $3 \times 3 \times 1$ super-cell for $2 \mathrm{H}-\mathrm{MoS}_{2}$ and a $3 \times 3 \times 2$ super-cell for $1 \mathrm{~T}-\mathrm{MoS}_{2}$ from their primary unit-cells, respectively. The two super-cells have equivalent Mo and S ions. By studying the change of relative energy of the two polytypes and the evolution of electronic structures around Fermi levels during $\mathrm{Li}$ ion intercalation, one can figure out the driving force for the $2 \mathrm{H}$-to-1T phase transition. Since $\mathrm{Li}$ ions can fill every vdW gap with a continuously variable Li content [54], we assume two principles for $\mathrm{Li}$ ion introductions, (1) each vdW gap may be intercalated with equal amount of Li ions, and (2) within each vdW gap. Li ion distribution should be as uniform as possible to maximize the $\mathrm{Li}-\mathrm{Li}$ interionic separation. Following these principles, the intercalated structure is expected to have the smallest Coulomb repulsion.

As indicated in Figure 1, there are two kinds of interstitial sites available for $\mathrm{Li}$ occupation. One is at octahedral sites and the other is at tetrahedral sites. Unlike the Li ion occupation at octahedral sites, the occupation of one $\mathrm{Li}$ ion at a tetrahedral site increases the lattice stress along the diagonal line direction of (110) face. As a result, the lattice can distort into a monoclinic shape and the $\mathrm{Li}$ ion is expelled out from the tetrahedral site. Therefore, to keep the configuration stable, two neighboring tetrahedral sites belonging to different interstitial layers within a vdW gap should be occupied simultaneously (i.e. forming a Li-ion pair). In this way, the stresses induced by the two Li ions cancel each other and the intercalated structure becomes stable.

To quantify the stability of Li intercalation at these possible sites, we define the formation energy of intercalated $\mathrm{Li}$ as $E_{\mathrm{f}}=E_{\mathrm{tot}}-E_{\mathrm{MoS} 2}-n E_{\mathrm{Li}}$. Here $E_{\mathrm{tot}}$ is the total energy of lithiated $\mathrm{MoS}_{2}, E_{\mathrm{MoS} 2}$ is the total energy of pure $\mathrm{MoS}_{2}$ host, $E_{\mathrm{Li}}$ is the total energy of body-centered cubic Li metal, and $n$ is the number of intercalated $\mathrm{Li}$ ions. If one $\mathrm{Li}$ ion is introduced at an octahedral site, the intercalated structure Li$\mathrm{MoS}_{2}$ forms. The calculated $E_{\mathrm{f}}$ is -1.593 and $-1.895 \mathrm{eV} / \mathrm{f}$.u. for $2 \mathrm{H}$ and $1 \mathrm{~T}$ phase respectively. If a Li-ion pair is introduced at two tetrahedral sites in the manner discussed above, $\mathrm{Li}_{2} \mathrm{MoS}_{2}$ may form. The calculated $E_{\mathrm{f}}$ of half the Li-ion pair (i.e. one tetrahedral interstitial $\mathrm{Li}$ ion) becomes -0.693 and $-0.636 \mathrm{eV} / \mathrm{f}$.u. for $2 \mathrm{H}$ and $1 \mathrm{~T}$ phase respectively. These calculations indicate that (1) octahedral sites are more favorable for $\mathrm{Li}$ ion intercalation than tetrahedral sites, regardless of whether the host phase is $2 \mathrm{H}$ or $1 \mathrm{~T}$; and (2) $\mathrm{Li}$ ion intercalation can be more easily realized in $1 \mathrm{~T}$ phase than in $2 \mathrm{H}$ phase. Therefore, the following studies will focus on $\mathrm{Li}$ ion occupation on octahedral sites.

By tracking different Li contents $(0,0.11,0.33,0.44$, $0.56,0.67,0.78$ and 1$)$, one gets the evolution of relative stability between $2 \mathrm{H}$ and $1 \mathrm{~T}$ in terms of the relative energy $E_{1 \mathrm{~T}}-E_{2 \mathrm{H}}$ (Figure 5). The total energy $E_{1 \mathrm{~T}}$ of $1 \mathrm{~T}-\mathrm{Li}_{x} \mathrm{MoS}{ }_{2}$ decreases with increasing $\mathrm{Li}$ content, indicating $1 \mathrm{~T}$ phase

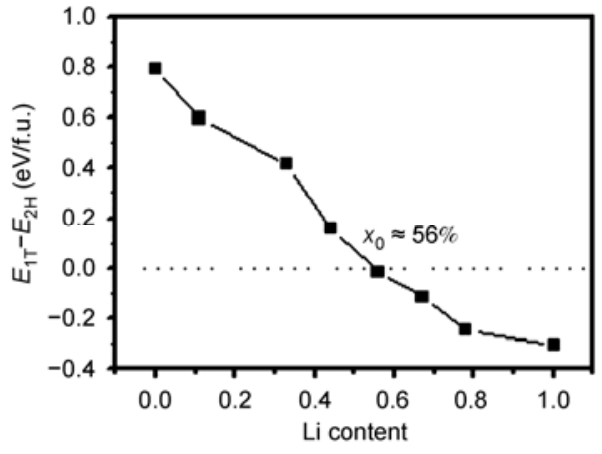

Figure 5 Li-content-dependent relative total energy of $1 \mathrm{~T}$ phase with respect to $2 \mathrm{H}$ phase. The total energy of $2 \mathrm{H}$ phase is set to be zero point. For Li content beyond $\sim 56 \%$, the total energy of $1 \mathrm{~T}$ phase is lower than that of $2 \mathrm{H}$ phase.

becomes energy favorable for $\mathrm{Li}$ intercalation. When $\mathrm{Li}$ percentage per $\mathrm{MoS}_{2}$ is beyond $\sim 56 \%, 1 \mathrm{~T}-\mathrm{Li}_{x} \mathrm{MoS}_{2}$ turns out to be more stable than $2 \mathrm{H}-\mathrm{Li}_{x} \mathrm{MoS}$, consistent with the experimental indication by Py et al. [28] and pointing to the falling of the first voltage plateau at $1.1 \mathrm{~V}$, which accounts for $\sim 60 \%$ capacity of fully lithiated $\mathrm{LiMoS}_{2}$. Details will be addressed in our future publication combined with our own experimental characterization.

Although possible structural disorder due to random $\mathrm{Li}$ occupation may slightly change electronic state at the bands near Fermi levels, a general trend with continuing electron injection into antibonding states is to weaken Mo-S $p$ - $d$ covalence interaction. This may bring two effects. First, the average Mo-S bond length is lengthened from $2.416 \AA$ in $2 \mathrm{H}-\mathrm{MoS}_{2}$ to $2.450 \AA$ in $2 \mathrm{H}-\mathrm{Li}_{x} \mathrm{MoS}_{2}(x=0.56)$. As a result, the crystal-field splitting is diminished and the crystal is destabilized. Second, since $p-d$ interaction is incompatible with center-inversion symmetry, the weakening of this interaction breaks the restriction of symmetry and enables the transition to the $D_{3 d}$ configuration. Another result of electron injection is that Mo $4 d$ electronic configuration changes from $d^{2}$ to $d^{3}$. The latter term has been found energetically favorable under $D_{3 d}$ group to stabilize crystal-field [55].

\subsection{Formation and stabilization of CDW after $\mathrm{Li}$ in- tercalation}

Li-intercalated normal $1 \mathrm{~T}$ phase is subject to the Peierls instability originating from Fermi surface nesting [33]. This is generally associated with two mechanisms: first, electrons are injected into previously unoccupied $d$-states, increasing the electron density on Mo and nesting the Fermi surface simultaneously, and second, the electron density is spatially modulated accompanied by a periodic lattice distortion (PLD) with complicated ionic patterns featured by reduced symmetry. Different from the electron self-modulation in such CDW materials as $\mathrm{NbSe}_{2}, \mathrm{TiSe}_{2}$ and $\mathrm{TaSe}_{2}$ [56-58], the modulation of electron density in Li-intercalated $\mathrm{MoS}_{2}$ requires outer electron injection and depends on the symmetry of host matrix $(1 \mathrm{~T}$ or $2 \mathrm{H})$. It is shown here that the 
Peierls instability exists also in the Li-intercalated $2 \mathrm{H}-\mathrm{MoS}_{2}$, which results in the formation of a CDW weaker than that in the $1 \mathrm{~T}$ counterpart.

Under the $D_{6 h}$ symmetry of $2 \mathrm{H}$ lattice, only the lowest conduction band $\alpha$ form nested Fermi surface, as seen from Figure 6(a) for $2 \mathrm{H}-\mathrm{LiMoS}_{2}$. Electrons brought in with $\mathrm{Li}$ ion are added on the antibonding channel constituted by $(p-d)^{*}$ state and $e^{\prime}$-band. The filling of $e^{\prime}$-band increases the electron density on Mo, while the filling of $(p-d)^{*}$ state leads to repelling between Mo and $\mathrm{S}$ which impedes Mo ion clustering. Since the hybridization of antibonding $\alpha$-band (by $d_{x y}$, $d_{x-y}^{2}$, and $d_{z}^{2}$ ) keeps approximately isotropic in the K- $\Gamma-\mathrm{M}$ plane, similar to the isotropic hybridization of the highest valence band in $2 \mathrm{H}-\mathrm{MoS}_{2}$ (Section 2.1), the Fermi surface nesting is moderate (Figure 6(c)). Therefore, a relatively small distortion is expected in $2 \mathrm{H}-\mathrm{LiMoS}_{2}$, resulting in the
CDW phase of $\mathrm{LiMoS}_{2}$ (denoted thereafter to be $\mathrm{C} 2 \mathrm{H}-$ $\mathrm{LiMoS}_{2}$ ).

To verify this conjecture, we examine a $(4 \times 4 \times 1) 2 \mathrm{H}-$ $\mathrm{LiMoS}_{2}$ supercell in which Mo planes are initially distorted according to the diamond pattern in C1T-LiMoS 2 [33]. This construction is reasonable because the nesting directions of $2 \mathrm{H}$ phase are the same as those of $1 \mathrm{~T}$ phase, as seen by comparison of Figure 6(c) and (d) (indicated by green arrows). In this way a strong initial Mo plane distortion is assumed that all the three nesting directions leads to Mo-Mo bonding. After careful structural relaxation, the original Mo clusters degenerate into zigzag chains with an average Mo-Mo bond length $2.92 \AA$, indicating that the initial distortion remains only in two directions. The derived $\mathrm{C} 2 \mathrm{H}-$ $\mathrm{LiMoS}_{2}$ has an orthorhombic lattice with a space group Pmma and point group $D_{2 h}$, as shown in Figure 6(e). The
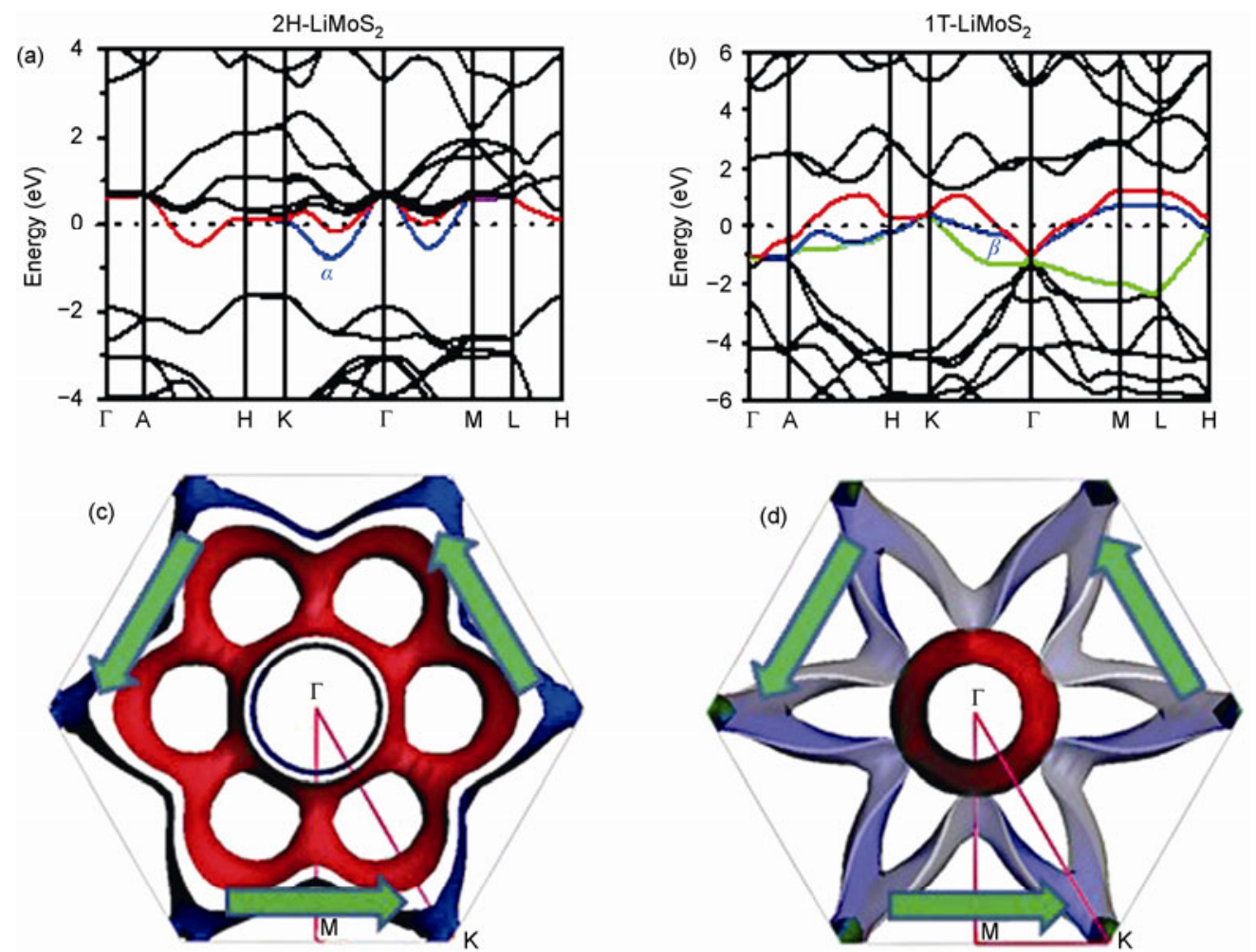

(e)

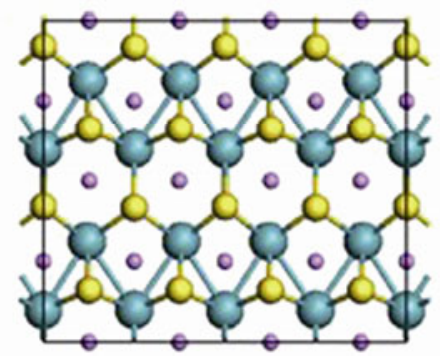

(f)

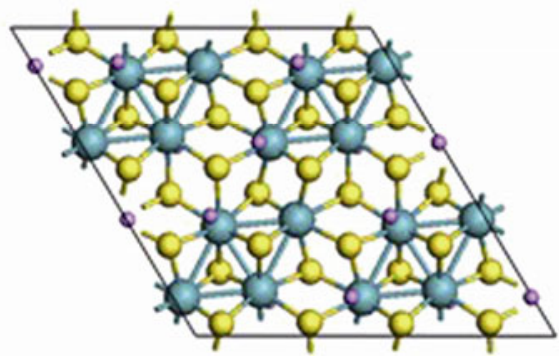

Figure 6 (a) and (b) are the band structures of $2 \mathrm{H}$ - and 1T-LiMoS 2 , respectively. The energy bands crossing the Fermi level are highlighted by colored lines. (c) and (d) show the Fermi surface profiles (from top view) of the bands with the corresponding colors in (a) and (b), respectively. For displaying the green regions which are covered by the blue region, the latter is drawn transparently in (d). Green arrows indicate three directions of nesting. (e) and (f) are top-view models of single-slab $\mathrm{C} 2 \mathrm{H}$ - and $\mathrm{C} 1 \mathrm{~T}-\mathrm{LiMoS}_{2}$, respectively. Mo ions are denoted by large green spheres. S ions are denoted by moderate yellow spheres and $\mathrm{Li}$ ions are denoted by small pink spheres. 
partial DOS of $\mathrm{C} 2 \mathrm{H}-\mathrm{LiMoS}_{2}$ in Figure 7(a) shows that $d$-state dominates around the Fermi level reflecting the effect of Mo clustering. However, this new phase is a metal with no gap opening but only a valley in the half-filled $e^{\prime}$ subband manifold because the CDW distortion is not strong enough to induce a strong electron localization. Note that a similar metal-to-metal CDW transition occurs in 1T-TiSe ${ }_{2}$ [59]. Additionally, Mo plane distortion with zigzag chains appears also in single-layer and restacked $\mathrm{MoS}_{2}[37,38]$ and $\mathrm{CDW}$ has been experimentally reported for $2 \mathrm{H}$-structured $\mathrm{Na}_{x} \mathrm{TaS}_{2}$ [60]. We have verified that the zigzag shape of Mo arrangement is maintained for smaller Li content down to $56 \%$.

The $2 \mathrm{H}$-to- $\mathrm{C} 2 \mathrm{H}$ transition results in an energy reduction of $\sim 0.15 \mathrm{eV} /$ f.u. for $\mathrm{LiMoS}_{2}$. Although smaller than that of $\sim 0.30 \mathrm{eV} / \mathrm{f} . \mathrm{u}$. induced by the transition to $1 \mathrm{~T}$ phase, the difference of energy reduction between the two transitions shrinks for smaller Li content. When Li content is $56 \%$, the energy reduction due to the $2 \mathrm{H}$-to- $\mathrm{C} 2 \mathrm{H}$ transition is $\sim 23$ meV/f.u., slightly larger than that induced by the $2 \mathrm{H}$-to- $1 \mathrm{~T}$ transition $(\sim 16 \mathrm{meV})$, indicating a possible competition between Mo plane distortion and $\mathrm{S}$ triangle rotation during $\mathrm{Li}$ ion intercalation. As a result, local disorder of arrangement and rotation may occur, perhaps corresponding to the observed amorphization after discharging at the $1.1 \mathrm{~V}$ voltage plateau [32].

Only when the transition to $1 \mathrm{~T}$ phase finishes, the further transition to C1T-LiMoS $S_{2}$ is possible. Under the $D_{3 d}$ symmetry of $1 \mathrm{~T}$ lattice, there is no bonding between $\mathrm{S}-p$ and Mo- $d$. The former is centralized around $\Gamma$ while the latter away from $\Gamma$ (Figure 3). Injected electrons will fill initially the S- $p$ state and then the Mo- $d$ state, leading to an increase of electron density on Mo sites finally. This is natural because injected electrons are more likely captured by electronegative atoms first. The absence of antibonding $(p-d)^{*}$ state in this case ensures a substantial increase of electron density on Mo.

Figure 6(b) and (d) shows that the Fermi surface nesting of $1 \mathrm{~T}-\mathrm{LiMoS}_{2}$ is mainly formed by the $\beta$ band (blue line). The hybridization of $\beta$ band is anisotropic in the $\mathrm{K}-\Gamma-\mathrm{M}$ plane, similar to the anisotropic hybridization of the highest valence band in $1 \mathrm{~T}-\mathrm{MoS}_{2}$ (Section 2.1). In the direction $\Gamma-\mathrm{K}$, band $\beta$ is featured mainly by $d_{x y}$, mixed with moderate $d_{y z}$ (not shown). While for $\Gamma-\mathrm{M}$, all five $d$ states are involved. As a result, $\beta_{\Gamma-\mathrm{K}}$ (i.e. band $\beta$ along $\Gamma-\mathrm{K}$ ) has relatively stronger electron localization than that of $\beta_{\Gamma-\mathrm{M}}$, reflected by the more flat dispersion of the former. Therefore, for a given Fermi energy much more $k$ points are involved in the direction $\Gamma$-K than in $\Gamma$-M, accounting for the strong nesting of Fermi surface (Figure 6(d)).

The real-space modulation of electron density is constrained within Mo planes because, first, all the nesting directions are parallel to Mo planes, and second, the major composition of $\beta_{\Gamma-\mathrm{K}}\left(d_{x y}\right)$ is within Mo planes. As a result,
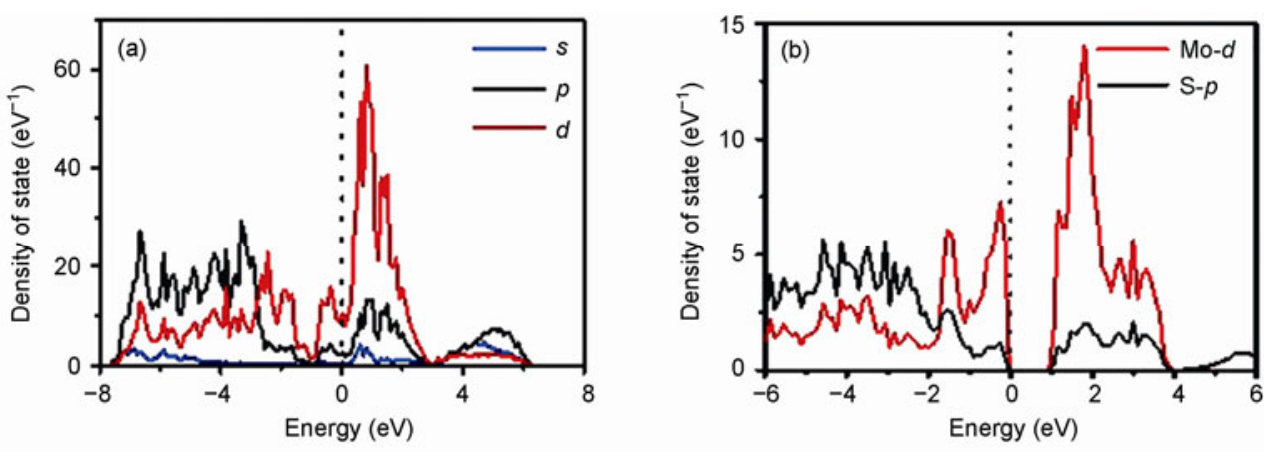

(c)

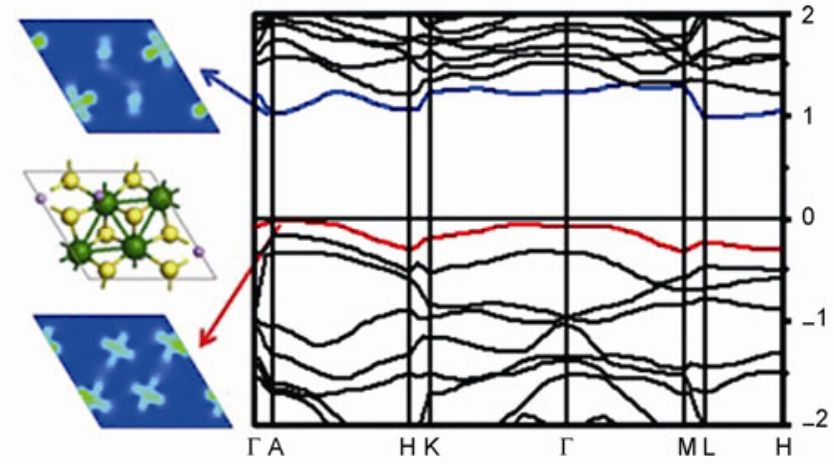

Figure 7 (a) and (b) are the partial density of states of C2H- and C1T-LiMoS 2 , respectively. For C1T-LiMoS 2 , only Mo- $d$ and S- $p$ are shown. (c) Band structures of relaxed $\mathrm{C} 1 \mathrm{~T}-\mathrm{LiMoS}_{2}$ along the $k$ point path shown in Figure 4. Partial charge density distribution corresponding to the valence band maximum and conduction band minimum are plotted and projected onto Mo planes. Top view of the primitive-cell model of $\mathrm{C}_{1 \mathrm{~T}}-\mathrm{LiMoS}$ is presented together. Mo ions are denoted by large green spheres. $\mathrm{S}$ ions are denoted by moderate yellow spheres and Li ions are denoted by small pink spheres. 
Mo ions cluster together to form diamond pattern, with an average bond length $2.93 \AA$ (Figure 6(f)). Since mainly $d$-band is filled in this stage, Mo-Mo bonding is enabled by increased $d$-electron interactions. We plot the energy bands of $\mathrm{C} 1 \mathrm{~T}-\mathrm{LiMoS}_{2}$ along the same $k$ point path as that of $1 \mathrm{~T}$ phase, although the $\mathrm{C} 1 \mathrm{~T}$ phase distorts slightly into a triclinic lattice. As shown in Figure 7(c), the C1T phase is an insulator having a large band gap of $\sim 1 \mathrm{eV}$. Both its edge bands present very flat dispersion, an indication of electron correlation enhancement, similar to that in $\mathrm{TiSe}_{2}$ [58]. The partial DOS in Figure 7(b) proves that those edge bands are dominated by Mo- $d$, indicating that the enhancement of electron correlation mainly comes from the $d$-band localization due to enhanced nearest-neighbor Mo-Mo $d$ - $d$ interaction. This is supported by the charge profiles representing Mo-Mo $d$ - $d$ interaction shown in Figure 7(c). It is seen that $d-d$ interaction leads to a bonding characteristic at VBM and an antibonding characteristic at CBM, similar to the case of $p$ - $d$ interaction in $2 \mathrm{H}-\mathrm{MoS}_{2}$.

The stabilization of CDW in Li-intercalated $\mathrm{MoS}_{2}$ (in both $2 \mathrm{H}$ and $1 \mathrm{~T}$ lattice) can be ascribed to the same nature of the Mott-Hubbard type transition [61,62]. However, the former is featured by two distinct characteristics. First, the enhancement of electron correlation is not on-site but comes from Mo-Mo $d$ - $d$ interaction, which results in electron localization within the domains formed by clustered Mo ions and prevents electron itinerating among the domains. This conforms to the extended Hubbard model which stresses the contribution of nearest-neighbor interaction [63]. Second, the magnitude of CDW in Li-intercalated $\mathrm{MoS}_{2}$ is affected by Mo-S $p$ - $d$ covalence interaction and Fermi surface nesting, which are governed by initial symmetry (trigonal prism or octahedron) and outer electron injection ( $\mathrm{Li}$ ion intercalation). For example, the strong Mo-S $p$ - $d$ covalence interaction due to the absence of inversion centers in $2 \mathrm{H}-\mathrm{LiMoS}_{2}$ weakens Mo-Mo $d$ - $d$ interaction and prevents electron localization, resulting in a moderate distortion and a zero or small band gap after the CDW transition, as is the case of the $2 \mathrm{H}$-to-C2H transition. The opposite applies when the undistorted phase has a vanished Mo-S $p$ - $d$ covalence interaction, which results in strong Mo clustering and a large band gap in the CDW phase, like the 1T-to-C1T transition. This argument also explains the similar phenomenon in group $\mathrm{V}$ dichalcogenides such as $\mathrm{NbSe}_{2}$ and $\mathrm{TaSe}_{2}$, i.e. the $\mathrm{CDW}$ in $1 \mathrm{~T}$ phase is stronger than that in $2 \mathrm{H}$ phase [13].

\section{Conclusion}

In summary, we use first-principles methods to study the mechanism of intercalation-induced phase transitions of $\mathrm{MoS}_{2}$. The inversion-symmetry-related Mo-S $p$ - $d$ interaction and the anisotropy of $d$-band hybridization are the critical factor influencing the structural phase transitions. We suggest that doping (e.g. iodine) and/or decreasing material size to nanometer scale may change electronic interaction and hybridization, and controls the structural phase transitions during charging and discharging. Li ion intercalation in $2 \mathrm{H}-\mathrm{MoS}_{2}$ leads to two competing effects, i.e. the transition to $1 \mathrm{~T}$ phase and the Mo plane distortion in $2 \mathrm{H}$ lattice. The Peierls instability exists in both $2 \mathrm{H}$ and $1 \mathrm{~T}$ hosts of Li-intercalated $\mathrm{MoS}_{2}$ while results in a stronger PLD in the latter. The stabilization of CDW in Li-intercalated $\mathrm{MoS}_{2}$ is related to the enhanced electron correlation due to Mo-Mo $d-d$ covalence interaction, which can be weakened by Mo-S $p$ - $d$ covalence interaction however. In $1 \mathrm{~T}$ phase of $\mathrm{Li}$ intercalated $\mathrm{MoS}_{2}$, the $d$-band nonbonding with $\mathrm{S}$ - $p$ ensures effective electron injection, and the hybridization anisotropy of $d$-band leads to strong Fermi surface nesting. Since the technological importance of $\mathrm{MoS}_{2}$ originates from its quasitwo-dimensional electronic structures, the insights gained in our work are helpful to the development of high-energydensity LIB with the band engineering as an important means to tailor the electrochemical performance.

The authors thank the helpful discussion with Guoqiang Liu. Shanghai Supercomputer Center is thanked for providing computation resources. This work was supported by the Ningbo Key Innovation Team and the Ningbo Natural Science Foundation (2011B82005, 2012A610098), the Natural Science Foundation of Zhejiang Province (LQ12A04004), the National Natural Science Foundation of China (11174301) and the National Basic Research Program of China (2012CB722700, SS2013AA050901). X. Chen appreciates supports by the Postdoctoral Foundation of China (2012M510156).

1 Radisavljevic B, Radenovic A, Brivio J, et al. Single-layer $\mathrm{MoS}_{2}$ transistors. Nat Nanotech, 2011, 6: 147-150

2 Martin C. Stepping up $\mathrm{MoS}_{2}$ integration. Nat Mater, 2012, 11: 829-829

3 Wang $\mathrm{H}$, Yu L, Lee Y H, et al. Integrated circuits based on bilayer $\mathrm{MoS}_{2}$ transistors. Nano Lett, 2012, 12: 4674-4680

4 Zeng H, Dai J, Yao W, et al. Valley polarization in $\mathrm{MoS}_{2}$ monolayers by optical pumping. Nat Nanotech, 2012, 7: 490-493

5 Mak K, Lee C, Hone J, et al. Atomically thin $\mathrm{MoS}_{2}$ : A new direct-gap semiconductor. Phys Rev Lett, 2010, 105: 136805

6 Splendiani A, Sun L, Zhang Y, et al. Emerging photoluminescence in monolayer $\mathrm{MoS}_{2}$. Nano Lett, 2010, 10: 1271-1275

7 Chen J, Kuriyama N, Yuan H, et al. Cheminform abstract: Electrochemical hydrogen storage in $\mathrm{MoS}_{2}$ nanotubes. J Am Chem Soc, 2002, 123: 11813-11814

8 Hinnemann B, Moses P G, Nørskov J K. Recent density functional studies of hydrodesulfurization catalysts: Insight into structure and mechanism. J Phys: Conden Matter, 2008, 20: 064236

9 Cheng F Y, Chen J, Gou X L. $\mathrm{MoS}_{2}-\mathrm{Ni}$ nanocomposites as catalysts for hydrodesulfurization of thiophene and thiophene derivatives. Adv Mater, 2006, 18: 2561-2564

10 Drummond C, Alcantar N, Israelachvili J, et al. Microtribology and friction-induced material transfer in $\mathrm{WS}_{2}$ nanoparticle additives. Adv Func Mater, 2001, 11: 348-354

11 Qi S K, Xue Q J, Zhang X S, et al. Oxidation and electron transfer on the rubbing surface of $\mathrm{MoS}_{2}$. Chin Sci Bull, 1995, 40: 300-302

12 Soon J M, Loh K P. Electrochemical double-layer capacitance of $\mathrm{MoS}_{2}$ nanowall films. Electrochem Solid-State Lett, 2007, 10: A250-A254

13 Friend R H, Yoffe A D. Electronic properties of intercalation complexes of the transition metal dichalcogenides. Adv Phys, 1987, 36: $1-94$

14 Julien C. Physical chemistry of lithium intercalation compounds. In: Julien C, Pereira-Ramos J P, Momchilov A, eds. Proceedings of the 
NATO Advanced Study Institute on New Trends in Intercalation Compounds for Energy Stoage. The Netherlands: Springer, 2002. 209-233

15 Chang K, Chen W. In situ synthesis of $\mathrm{MoS}_{2} /$ graphene nanosheet composites with extraordinarily high electrochemical performance for lithium ion batteries. Chem Commun, 2011, 47: 4252-4254

16 Chang K, Chen W. L-cysteine-assisted synthesis of layered $\mathrm{MoS}_{2} /$ graphene composites with excellent electrochemical performances for lithium ion batteries. ACS Nano, 2011, 5: 4720-4728

17 Xiao J, Choi D, Cosimbescu L, et al. Exfoliated $\mathrm{MoS}_{2}$ nanocomposite as an anode material for lithium ion batteries. Chem Mater, 2010, 22: $4522-4524$

18 Xiao J, Wang X, Yang X Q, et al. Electrochemically induced high capacity displacement reaction of $\mathrm{PEO} / \mathrm{MoS}_{2} /$ graphene nanocomposites with lithium. Adv Funct Mater, 2011, 21: 2840-2846

19 Winter M, Brodd R J. What are batteries, fuel cells, and supercapacitors? Chem Rev, 2004, 104: 4245-4270

20 Geim A K, Novoselov K S. The rise of graphene. Nat Mater, 2007, 6: 183-191

21 Yoo E, Kim J, Hosono E, et al. Large reversible Li storage of graphene nanosheet families for use in rechargeable lithium ion batteries. Nano Lett, 2008, 8: 2277-2282

22 Chang K, Chen W, Ma L, et al. Graphene-like $\mathrm{MoS}_{2} /$ amorphous carbon composites with high capacity and excellent stability as anode materials for lithium ion batteries. J Mater Chem, 2011, 21: 6251-6257

23 Feng C, Ma J, Li H, et al. Synthesis of molybdenum disulfide $\left(\mathrm{MoS}_{2}\right)$ for lithium ion battery applications. Mater Res Bull, 2009, 44: 18111815

24 Guo G, Hong J, Cong C, et al. Molybdenum disulfide synthesized by hydrothermal method as anode for lithium rechargeable batteries. J Mater Sci, 2005, 40: 2557-2559

25 Hwang H, Kim H, Cho J. $\mathrm{MoS}_{2}$ nanoplates consisting of disordered graphene-like layers for high rate lithium battery anode materials. Nano Lett, 2011, 11: 4826-4830

26 Zhu X, Chen N, Lian F, et al. First principle calculation of lithiation/ delithiation voltage in Li-ion battery materials. Chin Sci Bull, 2011, 56: 3229-3232

27 Chen X, He J, Srivastava D, et al. Electrochemical cycling reversibility of $\mathrm{LiMoS}_{2}$ using first-principles calculations. Appl Phys Lett, 2012, 100: 263901

28 Py M A, Haering R R. Structural destabilization induced by lithium intercalation in $\mathrm{MoS}_{2}$ and related compounds. Can J Phys, 1983, 61: $76-84$

29 Wypych F, Schollhorn R. 1T-MoS 2 , a new metallic modification of molybdenum disulfide. J Chem Soc Chem Commun, 1992, 1386-1388

30 Mulhern P J. Lithium intercalation in crystalline $\mathrm{Li}_{x} \mathrm{MoS}_{2}$. Can J Phys, 1989, 67: 1049-1052

31 Chrissafis K, Zamani M, Kambas K, et al. Structural studies of $\mathrm{MoS}_{2}$ intercalated by lithium. Mater Sci Eng B, 1989, 3: 145-151

32 Fang X, Hua C, Guo X, et al. Lithium storage in commercial $\mathrm{MoS}_{2}$ in different potential ranges. Electrochim Acta, 2012, 81: 155-160

33 Rocquefelte X, Boucher F, Gressier P, et al. Mo cluster formation in the intercalation compound $\mathrm{LiMoS}_{2}$. Phys Rev B, 2000, 62: 2397-2400

34 Rocquefelte X, Bouessay I, Boucher F, et al. Synergetic theoretical and experimental structure determination of nanocrystalline materials: Study of LiMoS 2 . J Solid State Chem, 2003, 175: 380-383

35 Petkov V, Billinge S J L, Larson P, et al. Structure of nanocrystalline materials using atomic pair distribution function analysis: Study of LiMoS $_{2}$. Phys Rev B, 2002, 65: 092105

36 Dungey K E, Curtis M D, Penner-Hahn J E. Structural characterization and thermal stability of $\mathrm{MoS}_{2}$ intercalation compounds. Chem Mater, 1998, 10: 2152-2161

37 Gordon R A, Yang D, Crozier E D, et al. Structures of exfoliated single layers of $\mathrm{WS}_{2}, \mathrm{MoS}_{2}$, and $\mathrm{MoSe}_{2}$ in aqueous suspension. Phys Rev B, 2002, 65: 125407
38 Heising J, Kanatzidis M G. Structure of restacked $\mathrm{MoS}_{2}$ and $\mathrm{WS}_{2}$ elucidated by electron crystallography. J Am Chem Soc, 1999, 121: 638-643

39 Mattheiss L F. Band structures of transition-metal-dichalcogenide layer compounds. Phys Rev B, 1973, 8: 3719-3740

40 Enyashin A, Gemming S, Seifert G. Nanosized allotropes of molybdenum disulfide. Eur Phys J Special Topics, 2007, 149: 103-125

41 Bromley R A. A semi-empirical tight-binding calculation of the band structure of $\mathrm{MoS}_{2}$. Phys Lett A, 1970, 33: 242-243

42 McMenamin J C, Spicer W E. Photoemission studies of layered transition-metal dichalcogenides: $\mathrm{MoS}_{2}$. Phys Rev B, 1977, 16: 5474-5487

43 Coehoorn R, Haas C, de Groot R A. Electronic structure of $\mathrm{MoSe}_{2}$, $\mathrm{MoS}_{2}$, and $\mathrm{WSe}_{2}$. II. The nature of the optical band gaps. Phys Rev B, 1987, 35: 6203-6206

44 Raybaud P, Hafner J, Kresse G, et al. Ab initio density functional studies of transition-metal sulphides: II. Electronic structure. J Phys: Conden Matter, 1997, 9: 11107-11140

45 Wilson J A, Yoffe A D. The transition metal dichalcogenides discussion and interpretation of the observed optical, electrical and structural properties. Adv Phys, 1969, 18: 193-335

46 Reshak A H, Auluck S. Calculated optical properties of $2 \mathrm{H}-\mathrm{MoS}_{2}$ intercalated with lithium. Phys Rev B, 2003, 68: 125101-125107

47 Schönfeld B, Huang J J, Moss S C. Anisotropic mean-square displacements (MSD) in single-crystals of $2 \mathrm{H}-$ and $3 \mathrm{R}-\mathrm{MoS}_{2}$. Acta Cryst B, 1983, 39: 404-407

48 Kresse G, Furthmüller J. Efficient iterative schemes for ab initio total-energy calculations using a plane-wave basis set. Phys Rev B, 1996, 54: 11169-11186

49 Blöchl P E. Projector augmented-wave method. Phys Rev B, 1994, 50: $17953-17979$

50 Grimme S. Semiempirical gga-type density functional constructed with a long-range dispersion correction. J Comput Chem, 2006, 27: 1787-1799

51 Kugler H K. Gmelin Handbook of Inorganic and Organometallic Chemistry. Dordrecht: Springer, 1995. B7

52 Reshak A H, Auluck S. Band structure and optical response of $2 \mathrm{H}-$ $\mathrm{MoX}_{2}$ compounds (X=S, Se, and Te). Phys Rev B, 2005, 71: 155114

53 Kohn W, Sham L J. Self-consistent equations including exchange and correlation effects. Phys Rev, 1965, 140: A1133-A1138

54 Persson K, Hinuma Y, Meng Y S, et al. Thermodynamic and kinetic properties of the $\mathrm{Li}$-graphite system from first-principles calculations. Phys Rev B, 2010, 82: 125416

55 Huisman R, de Jonge R, Haas C, et al. Trigonal-prismatic coordination in solid compounds of transition metals. J Solid State Chem, 1971, 3: 56-66

56 Horiba $\mathrm{K}$, Ono $\mathrm{K}$, Oh J H, et al. Charge-density wave and threedimensional fermi surface in $1 \mathrm{~T}-\mathrm{TaSe}_{2}$ studied by photoemission spectroscopy. Phys Rev B, 2002, 66: 073106

57 Tonjes W C, Greanya V A, Liu R, et al. Charge-density-wave mechanism in the $2 \mathrm{H}-\mathrm{NbSe}_{2}$ family: Angle-resolved photoemission studies. Phys Rev B, 2001, 63: 235101

58 Zhu Z, Cheng Y, Schwingenschlögl U. Origin of the charge density wave in 1T-TiSe 2 . Phys Rev B, 2012, 85: 245133

$59 \mathrm{Li} \mathrm{G}, \mathrm{Hu}$ W Z, Qian D, et al. Semimetal-to-semimetal charge density wave transition in 1T-TiSe 2 . Phys Rev Lett, 2007, 99: 027404

60 Shen D W, Xie B P, Zhao J F, et al. Novel mechanism of a charge density wave in a transition metal dichalcogenide. Phys Rev Lett, 2007, 99: 216404

61 Mott N F, Peierls R. Discussion of the paper by de boer and verwey. Proc Phys Soc, 1937, 49: 72-73

62 Mott N F. The basis of the electron theory of metals, with special reference to the transition metals. Proc Phys Soc A, 1949, 62: 416-422

63 van Dongen P G J. Extended hubbard model at strong coupling. Phys Rev B, 1994, 49: 7904-7915

Open Access This article is distributed under the terms of the Creative Commons Attribution License which permits any use, distribution, and reproduction in any medium, provided the original author(s) and source are credited. 